\title{
The 14th International Symposium on Alcoholic Liver and Pancreatic Diseases and Cirrhosis (ISALPDC)
}

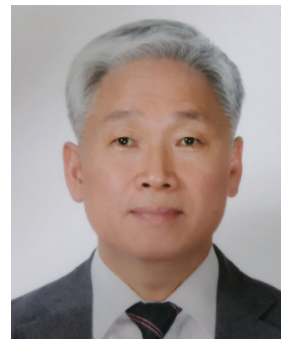

Dong Joon Kim

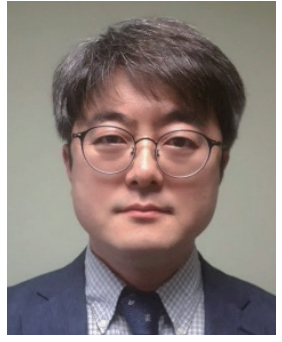

Won-II Jeong

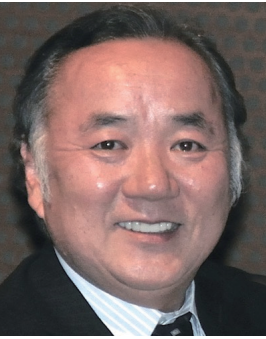

Hidekazu Tsukamoto

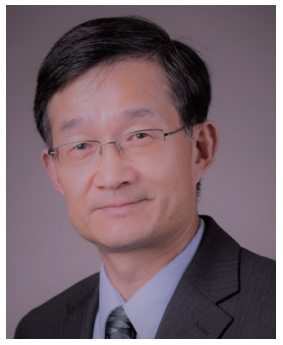

Bin Gao
Dear Colleagues,

The whole world has been in trouble with COVID-19. However, in the evolution of this pandemic, human has never ceased making efforts for medical and research advancements in the midst of difficulties.

We were delighted to host the virtual 14th International Symposium on Alcoholic Liver and Pancreatic Diseases and Cirrhosis (ISALPDC) in Seoul, Korea, on August 13-14, 2020. The ISALPDC was held in conjunction with The Liver Week 2020, an international conference hosted by the Korean Association for the Study of the Liver (KASL) and co-hosted by the Korean Association of HBP Surgery (KAHBPS), the Korean Liver Cancer Association (KLCA) and the Korean Liver Transplantation Society (KLTS).

Alcohol misuse is a major contributor to injury, mortality, and the burden of disease globally. In 2016, approximately 3.0 million deaths worldwide were attributable to alcohol, responsible for $5.3 \%$ of all deaths. These facts are the basis of the global outreach objective pursued by the ISALPDC.

We are honored to have the opening remarks jointly delivered by Dr. George Koob, Director of the National Institutes on Alcohol Abuse and Alcoholism (NIAAA)/National Institutes of Health (NIH) and Dr. Katherine Jung, Director, Division of Metabolism and Health Effects of NIAAA. The symposium showcased 17 renowned speakers and 6 young investigator awardees and was attended by more than 1,455 participants from 28 countries to discuss the cutting-edge research on obesity-alcohol synergism, novel cellular and organ crosstalk in alcoholic liver disease (ALD), alcohol promotion of cancer, and translational studies and public health on ALD.

This symposium was supported by the KASL, a grant from NIAAA (5R13AA020691), Southern California Research Center for ALPD and Cirrhosis (P50AA011999), and other sponsors. We cordially appreciate their support. We also thank all speakers, moderators, and attendees who have made the symposium most enlightening and fruitful.

This special issue represents an outstanding compendium of review articles on topics covered by the symposium speakers and two keynote speakers of the Liver Week 2020, Drs. Gyongyi Szabo and Bernd Schnabl. We sincerely thank them for the invaluable contributions. Finally, we wish to express our sincere gratitude to the organizing committee members of the Liver Week 2020 and the editorial committee of Clinical and Molecular Hepatology for their understanding and support. 


\section{Dong Joon Kim}

Professor, Hallym University College of Medicine

Director, Institute for Liver and Digestive Disease, Hallym University

\section{Won-Il Jeong}

Professor, Laboratory of Liver Research, KAIST, Daejeon

\section{Hidekazu Tsukamoto}

Professor of Pathology

Director, Southern California Research Center for ALPD and Cirrhosis

University of Southern California, Los Angeles

\section{Bin Gao}

Chief, Laboratory of Liver Diseases, NIAAA/NIH, Bethesda 